\title{
Queda livre de duas massas acopladas por uma mola em um campo gravitacional uniforme
}

Free fall of two masses coupled by a spring in a uniform gravitational field

\author{
A. C. Tort ${ }^{* 10}$, M. F. B. Francisquini ${ }^{2}$, R. R. Machado ${ }^{3}$ \\ ${ }^{1}$ Universidade Federal do Rio de Janeiro, Instituto de Física, Rio de Janeiro, RJ, Brasil. \\ ${ }^{2}$ Instituto Federal de Educação, Ciência e Tecnologia do Rio de Janeiro, Niterói, RJ, Brasil. \\ ${ }^{3}$ Centro Federal de Educação Tecnológica Celso Suckow da Fonseca, Rio de Janeiro, RJ, Brasil.
}

\begin{abstract}
Recebido em 01 de outubro de 2020. Revisado em 28 de novembro de 2020. Aceito em 01 de dezembro de 2020.
Quando nos deparamos com problemas de mecânica, muitas vezes somos guiados por um senso de familiaridade e de analogias com situações prévias. No entanto, esta nem sempre parece ser uma boa alternativa. Ao contrário, há situações em que a simples analogia com situações parecidas nos conduz à resposta errada e a demonstração experimental se faz necessária para o entendimento do problema. Apresentamos neste trabalho uma destas situações. em que a falta de conhecimento sobre o comportamento de queda de um sistema massa-mola pode ser o motivo da dificuldade de alunos de nível médio e superior acerca da situação proposta.
\end{abstract}

Palavras-chave: Ensino de física, sistemas massa-mola, queda-livre.

In solving classical mechanics problems many times we are guided by a feeling of familiarity and analogies. Nevertheless, not always this a good strategy. This is the case of the problem of two masses in free fall in a uniform field which we discuss in this paper.

Keywords: Physics teaching, mass-spring systems; free fall.

\section{Introdução}

Uma característica comum ao raciocínio físico é a presença de analogias para responder perguntas e formular hipóteses. Este artifício é particularmente útil quando a partir de uma situação conhecida tentamos descrever uma outra que não nos é familiar. Se realizado de maneira indiscriminada, no entanto, este mecanismo acaba conduzindo o aluno, e mesmo professores, a erros que nem sempre sabemos a que atribuir. O fato é que sendo a física uma ciência empírica, não podemos prescindir dos aspectos fenomenológicos imbricados nela e, neste sentido, recorrer à demonstração experimental nos parece ser a melhor alternativa, quando ela assim estiver disponível. Um exemplo fascinante é o da queda livre de duas massas acopladas por uma mola em um campo gravitacional uniforme. O problema é apresentado e discutido por Lang da Silveira e Axt que utilizaram técnicas fotográficas e análise das imagens obtidas para mostrar que sob certas condições é possível chegar a resultados à primeira vista surpreendentes [1] .

No presente trabalho, discutiremos um modelo teórico simples para a configuração utilizada em [1] que nos permitirá reproduzir se não todos, pelo menos alguns dos aspectos principais do experimento.

\footnotetext{
* Endereço de correspondência: tort@if.ufrj.br
}

\section{A Formulação do Problema}

Imaginemos um sistema formado por dois blocos idênticos de massa $m$ que denotaremos por 1 e 2, respectivamente, ligados por uma mola ideal no primeiro caso e por um no fio segundo, veja as Figuras 1(a) e 1 (b). Além disso, nas duas configurações, o bloco 1 está ligado ao teto por um fio ideal. Será que poderíamos, a partir destas configurações iniciais, estimar as acelerações de ambos os blocos imediatamente após cortarmos o fio que liga o bloco 1 ao teto? Em um primeiro momento, é comum que os alunos estabeleçam uma relação direta entre os dois casos e concluam que nas duas situações os blocos caiam com aceleração g, mas como veremos a solução não é tão simples assim.

No caso em que os blocos estão ligados por um fio, a situação de equilíbrio é trivialmente descrita pela identificação das forças, Figura 2 e pelas equações que descrevem o equilíbrio estático:

$$
\mathbf{T}_{1}=-\left(\mathbf{P}_{1}+\mathbf{T}\right)
$$

e

$$
\mathbf{T}=\mathbf{P}_{2}
$$

Ao rompermos o fio que liga o bloco 1 ao teto, fazemos com que o fio entre os blocos 1 e 2 deixe de ficar tensionado, isto é: eliminamos o vínculo entre os corpos, de modo que as únicas forças que agem sobre os blocos 1 


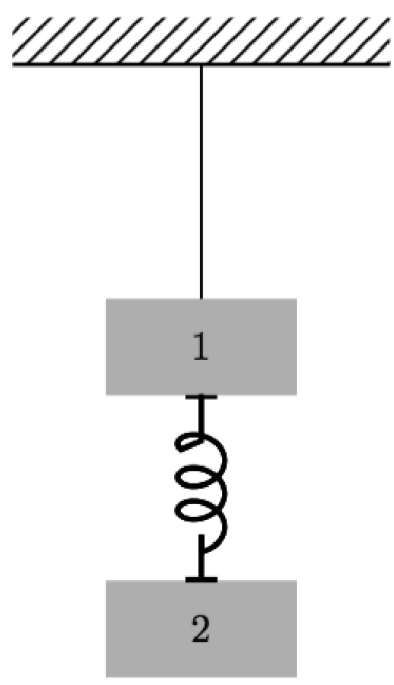

(a)

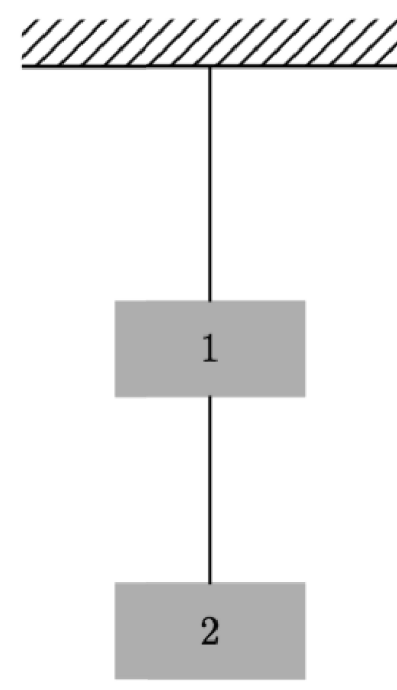

(b)
Figura 1: (a) Configuração inicial de duas massas unidas por uma mola de massa desprezível e (b) um fio ideal.
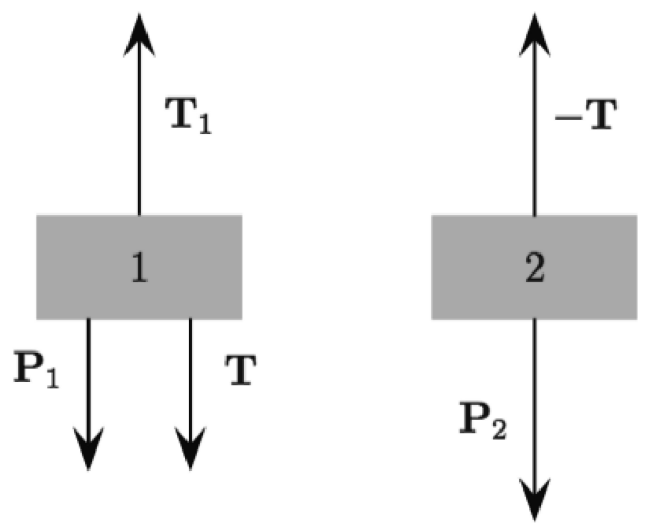

Figura 2: Diagramas das forças que atuam sobre cada uma das massas no caso em que estas estão unidas for um fio ideal.

e 2 nesta situação são seus respectivos pesos. As equações de movimento são:

$$
m \mathbf{a}_{1}=m \mathbf{g} ; \quad m \mathbf{a}_{2}=m \mathbf{g} .
$$

Portanto, os blocos caem com a mesma aceleração g. Se o fio que liga os dois blocos não fosse de massa desprezível como supusemos aqui, mas ainda assim inextensível, também estaria submetido apenas ao seu próprio peso caindo junto com os blocos com a aceleração $\mathbf{g}$.

Vejamos agora o que acontece no caso em que os dois blocos estão ligados por uma mola de massa desprezível. Analisando a Figura 3 no equilíbrio, as equações teriam uma forma semelhante às anteriores:

$$
\mathbf{T}_{1}=-\left(\mathbf{P}_{1}+\mathbf{F}_{\mathrm{el}}\right)
$$

e

$$
\mathbf{F}_{\mathrm{el}}=\mathbf{P}_{2}
$$
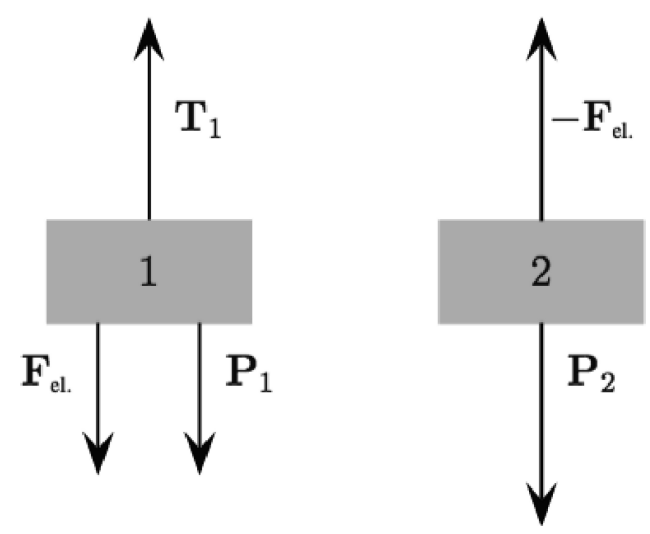

Figura 3: Sistema de blocos ligados por uma mola.

Ao rompermos o fio que liga o bloco 1 ao teto, desta vez, não eliminamos o vínculo entre os corpos, por este motivo, podemos rescrever as equações de movimento

$$
\mathbf{F}_{1 \text { res }}=\mathbf{P}_{1}+\mathbf{F}_{\mathrm{el}}
$$

para o primeiro corpo, e para o segundo corpo

$$
\mathbf{F}_{2 \text { res }}=\mathbf{P}_{2}-\mathbf{F}_{\mathrm{el}} \text {. }
$$

Mas imediatamente após o rompimento do fio que une o bloco 1 ao suporte fixo $\mathbf{F}_{2 \text { res }}=0, \operatorname{logo}$, a força resultante sobre o primeiro corpo, $\mathbf{F}_{1 \text { res. }}$, pode ser escrita como

$$
\mathbf{F}_{1 \text { res. }}=2 \mathbf{P}_{1} \text {, }
$$

ou ainda

$$
\mathbf{F}_{1 \text { res. }}=2 m \mathbf{g},
$$

ou seja, a aceleração do corpo 1 imediatamente após o rompimento do fio que o liga ao teto é igual a duas vezes a aceleração da gravidade, enquanto, neste mesmo instante, a aceleração do corpo 2 é igual a 0 . Isto nos permite inferir que, no momento em que o bloco 1 é verticalmente acelerado para baixo com $2 \mathrm{~g}$, o bloco 2 permanece estático no ar.

\section{Solução para o Caso de Massas Iguais}

Como exposto até aqui, o problema pode ser discutido com alunos do ensino médio mais interessados e nos cursos de mecânica básica no nível universitário. Entretanto, é possível avançar um pouco mais e obter uma solução para qualquer instante de tempo $t \geq 0$.

Seja $L_{0}$ o comprimento de repouso da mola e consideremos o eixo vertical $y$ positivo na direção e sentido de $\mathbf{g}$, veja a Figura 4 As equações de movimento dos blocos são

$$
m \frac{d^{2} y_{1}}{d t^{2}}=+\kappa\left(y_{2}-y_{1}-L_{0}\right)+m g
$$

e

$$
m \frac{d^{2} y_{2}}{d t^{2}}=-\kappa\left(y_{2}-y_{1}-L_{0}\right)+m g
$$




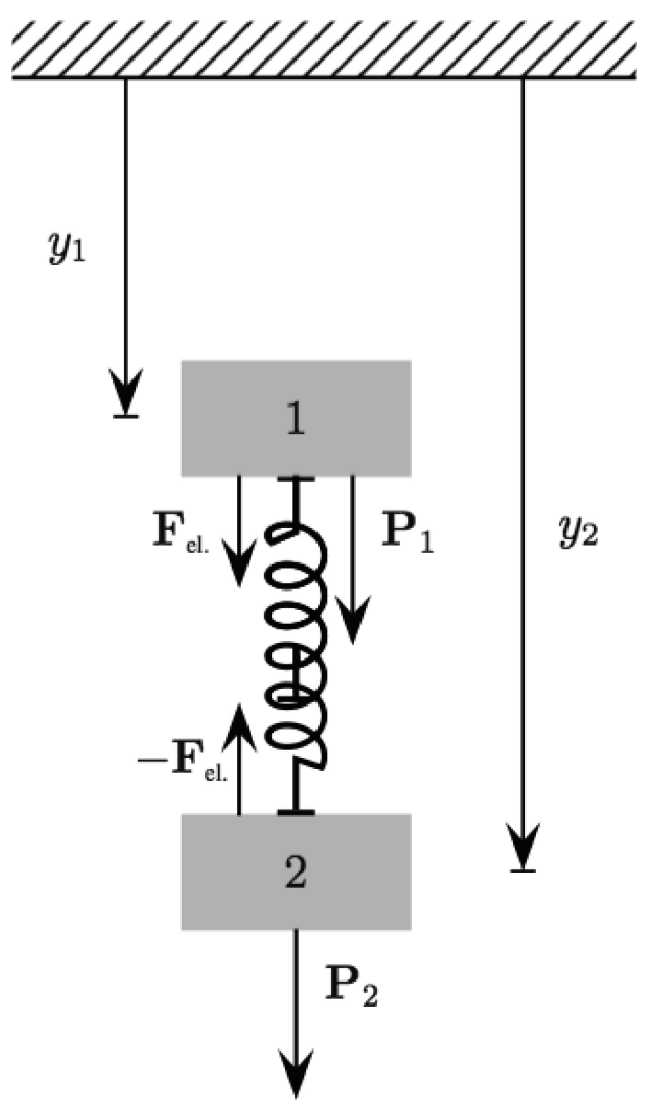

Figura 4: Corpos de massas iguais em queda unidos por uma mola de massa desprezível.

Para desacoplar as equações de movimento, convém introduzir duas novas variáveis definidas pelas seguintes relações

$$
\eta=\frac{y_{1}+y_{2}}{2}
$$

$\mathrm{e}$

$$
\zeta=y_{2}-y_{1}
$$

A primeira é simplesmente a posição do centro de massa dos dois blocos e a segunda, a posição relativa. Com estas novas variáveis, as equações (10) e 11) podem ser rescritas na forma:

$$
\frac{d^{2} \eta}{d t^{2}}=g
$$

$\mathrm{e}$

$$
m \frac{d^{2} \zeta}{d t^{2}}=-2 \kappa \zeta+\frac{2 \kappa L_{0}}{2}
$$

Ou ainda

$$
\frac{d^{2} \zeta}{d t^{2}}+\omega^{2} \zeta=2 \kappa L_{0}
$$

onde $\omega^{2}=\frac{2 \kappa}{m}$. A soluções das equações 14 e 16 podem ser facilmente obtidas. A solução da equação 14 é dada por

$$
\eta=A+B t+\frac{g t^{2}}{2}
$$

onde $A$ e $B$ são constantes que devem ser determinadas pelas condições iniciais. Esta solução mostra-nos que o centro de massa dos blocos acoplados está em quedalivre com aceleração $g$.

A solução da equação (16) é dada pela combinação linear da solução da equação homogênea com uma solução particular. É fácil ver que a solução particular é o comprimento relaxado da molla, $L_{0}$. Portanto, escrevemos:

$$
\zeta=C \cos (\omega t)+D \sin (\omega t)+L_{0},
$$

onde $C$ e $D$ são constantes a serem determinadas pelas condições inciais.

Por simplicidade, escolhemos a origem do sistema de coordenadas na posição inicial do centro de massa. Admitiremos também que as velocidades iniciais dos blocos são nulas, logo,

$$
\begin{aligned}
y_{1}(0) & =-\frac{L_{0}}{2}+\frac{m g}{2 \kappa}, & \frac{d y_{1}(0)}{d t}=0 \\
y_{2}(0) & =\frac{L_{0}}{2}+\frac{m g}{2 \kappa} ; & \frac{d y_{2}(0)}{d t}=0 .
\end{aligned}
$$

Traduzidas para a variável $\eta$ e aplicadas as condições iniciais abaixo

$$
\eta(0)=0
$$

$\mathrm{e}$

$$
\frac{d \eta(0)}{d t}=0
$$

segue que

$$
\eta=\frac{g t^{2}}{2}
$$

Para a variável $\zeta$, as condições iniciais são:

$$
\zeta(0)=L_{0}+\frac{m g}{\kappa}
$$

e

$$
\frac{d \zeta(0)}{d t}=0
$$

$\log$,

$$
\zeta=\left(\frac{m g}{\kappa}\right) \cos (\omega t)+L_{0} .
$$

Podemos facilmente voltar para as variáveis originais e escrever as soluções em termos de $y_{1}$ e $y_{2}$ :

$$
y_{1}=\frac{g t^{2}}{2}-\left(\frac{m g}{2 \kappa}\right) \cos (\omega t)-\frac{L_{0}}{2},
$$


e

$$
y_{2}=\frac{g t^{2}}{2}+\left(\frac{m g}{2 \kappa}\right) \cos (\omega t)+\frac{L_{0}}{2} .
$$

Se agora calcularmos as acelerações dos blocos obteremos:

$$
a_{1}=g(1+\cos \omega t) ; \quad a_{2}=g(1-\cos \omega t) !
$$

Fazendo $t=0$ obtemos $a_{1}=2 g$ e $a_{2}=0$, de acordo com a nossa análise semi-qualitativa inicial.

\section{Solução para o Caso de Massas Diferentes}

Podemos analisar o problema agora admitindo massas diferentes. Dessa forma, podemos generalizar a discussão anterior e resolver casos extremos como fazendo uma massa muito maior do que a outra. A modelagem é análoga à feita com as equações 100 e 11

$$
m_{1} \frac{d^{2} y_{1}}{d t^{2}}=+\kappa\left(y_{2}-y_{1}-L_{0}\right)+m_{1} g
$$

$$
m_{2} \frac{d^{2} y_{2}}{d t^{2}}=-\kappa\left(y_{2}-y_{1}-L_{0}\right)+m_{2} g
$$

Introduzindo uma mudança de variáveis podemos desacoplar as equações acima. Para isso, utilizaremos a equação para o movimento do centro de massa e a coordenada relativa $\left(y_{2}-y_{1}\right)$, isto é:

$$
\eta=\frac{m_{1} y_{1}+m_{2} y_{2}}{m_{1}+m_{2}}
$$

e

$$
\zeta=y_{2}-y_{1} .
$$

Dividindo a equação 30 por $m_{1}$ e a equação 31 por $m_{2}$ e subtraindo uma da outra obtemos:

$$
\frac{d^{2} y_{2}}{d t^{2}}-\frac{d^{2} y_{1}}{d t^{2}}=\left(\frac{1}{m_{1}}+\frac{1}{m_{2}}\right) \kappa\left(y_{2}-y_{1}-L_{0}\right),
$$

que pode ser rescrita na forma:

$$
\frac{d^{2} \zeta}{d t^{2}}=-\frac{\kappa}{\mu}\left(\zeta-L_{0}\right)
$$

onde $\mu=\frac{m_{1} m_{2}}{m_{1}+m_{2}}$ é a massa reduzida do sistema. A equação para o movimento do centro de massa pode ser escrita facilmente:

$$
\frac{d^{2} \eta}{d t^{2}}=g
$$

As soluções das equações (35) e (36) são bem conhecidas. A solução da equação 36 é dada por

$$
\eta=A+B t+\frac{g t^{2}}{2}
$$

onde $A$ e $B$ são constantes determinadas pelas condições iniciais. A solução da equação (35) é dada pela solução da equação homogênea linearmente combinada com a solução particular que no caso é simplesmente uma constante, logo

$$
\zeta=C \cos \left(\omega^{\prime} t\right)+D \sin \left(\omega^{\prime} t\right)+L_{0},
$$

onde $\mathrm{C}$ e D são constantes a serem determinadas pelas condições inciais, $L_{0}$ representa o comprimento relaxado da mola e $\omega^{\prime}=\sqrt{\frac{\kappa}{\mu}}$. Escolhendo como origem do sistema de coordenadas a posição inicial do centro de massa e supondo que as velocidades iniciais dos blocos são nulas, teremos,

$$
\begin{gathered}
y_{1}(0)=-\left(\frac{m_{2}}{m_{1}+m_{2}}\right)\left(L_{0}+\frac{m_{2} g}{\kappa}\right) ; \quad \frac{d y_{1}(0)}{d t}=0 ; \\
y_{2}(0)=\left(\frac{m_{1}}{m_{1}+m_{2}}\right)\left(L_{0}+\frac{m_{2} g}{\kappa}\right) ; \quad \frac{d y_{2}(0)}{d t}=0 .
\end{gathered}
$$

Portanto, as condições inciais para a variável $\eta$ são

$$
\eta(0)=0,
$$

$\mathrm{e}$

$$
\frac{d \eta(0)}{d t}=0
$$

Fazendo uso das condições iniciais estabelecidas acima temos

$$
\eta=\frac{g t^{2}}{2}
$$

Aplicando as condições iniciais à variável $\zeta$, temos

$$
\zeta(0)=L_{0}+\frac{m_{2} g}{\kappa},
$$

$$
\frac{d \zeta(0)}{d t}=0
$$

A solução é imediata e se escreve:

$$
\zeta=\left(\frac{m_{2} g}{\kappa}\right) \cos \left(\omega^{\prime} t\right)+L_{0} .
$$

Podemos facilmente reescrever as soluções em termos de $y_{1}$ e $y_{2}$ a partir das seguintes transformações

$$
y_{1}=\eta-\frac{m_{2}}{m_{1}+m_{2}} \zeta,
$$

$$
y_{2}=\eta+\frac{m_{1}}{m_{1}+m_{2}} \zeta
$$

Portanto,

$$
y_{1}=\frac{g t^{2}}{2}-\left[\frac{m_{2}^{2} g}{\left(m_{1}+m_{2}\right) \kappa}\right] \cos \left(\omega^{\prime} t\right)-\frac{m_{2}}{m_{1}+m_{2}} L_{0}
$$


e

$$
y_{2}=\frac{g t^{2}}{2}+\left[\frac{m_{2} m_{1} g}{\left(m_{1}+m_{2}\right) \kappa}\right] \cos \left(\omega^{\prime} t\right)+\frac{m_{1}}{m_{1}+m_{2}} L_{0} .
$$

As velocidades das massas 1 e 2 são dadas por

$$
\begin{aligned}
& \dot{y}_{1} \equiv v_{1}=g t+\frac{m_{2}^{2} g}{m_{1}+m_{2}} \frac{\omega^{\prime}}{\kappa} \sin \left(\omega^{\prime} t\right) ; \\
& \dot{y}_{2} \equiv v_{2}=g t-\frac{m_{2}^{2} g}{m_{1}+m_{2}} \frac{\omega^{\prime}}{\kappa} \sin \omega^{\prime} t ;
\end{aligned}
$$

e as acelerações são dadas por

$$
\begin{aligned}
& \ddot{y}_{1} \equiv a_{1}=g+\frac{m_{2}^{2} g}{m_{1}+m_{2}} \frac{\omega^{\prime 2}}{\kappa} \cos \left(\omega^{\prime} t\right) ; \\
& \ddot{y}_{2} \equiv a_{2}=g-\frac{m_{2} m_{1} g}{m_{1}+m_{2}} \frac{\omega^{\prime 2}}{\kappa} \cos \left(\omega^{\prime} t\right) .
\end{aligned}
$$

Lembrando que $\omega^{\prime 2}=\frac{\kappa}{\mu}$, onde $\mu=\frac{m_{1} m_{2}}{m_{1}+m_{2}}$, e que por definição a aceleração do centro de massa é dada por:

$$
a_{\text {c. de } \mathrm{m} .}=\frac{m_{1} a_{1}+m_{2} a_{2}}{m_{1}+m_{2}},
$$

podemos verificar facilmente que

$$
a_{\text {c. de m. }}=g \text {, }
$$

isto é: o centro de massa está em queda livre. Podemos ver também que as acelerações dos blocos no instante inicial $t=0$ serão dadas por:

$$
a_{1}(t=0)=\frac{m_{1}+m_{2}}{m_{1}} g
$$

$\mathrm{e}$

$$
a_{2}(t=0)=0 \text {. }
$$

Estes resultados mostram-nos que independentemente do valor das massas, a aceleração do bloco inferior (bloco 2) no instante inicial será sempre nula. Por outro lado, se as massas são iguais, obtemos para $a_{y 1}(t=0)=$ $2 g$ como antes. Outro resultado interessante é o do caso em que $m_{2} \gg m_{1}$. Segue que no instante inicial:

$$
a_{1}(t=0) \approx \frac{m_{2}}{m_{1}} g .
$$

Se a razão $\frac{m_{2}}{m_{1}} \approx 40$, estaremos em acordo com Lang da Silveira e Axt quando afirmam que sob certas condições, a aceleração da extremidade superior pode ser estimada em até quarenta vezes a aceleração da gravidade [1].

\section{Comentários Finais}

O exemplo discutido aqui é uma excelente oportunidade de mostrar explicitamente aos nossos alunos como experimento e teoria podem andar de mãos dadas, mesmo que o modelo teórico seja uma aproximação ao sistema físico real que estamos estudando. Observemos também que este exemplo com as devidas omissões pode ser discutido no ensino médio - seções 1 e 2 combinadas com a referência [1] - e no ensino universitário - seção 3, apropriada para um curso de mecânica introdutória, e 4, mais adequada para um curso de mecânica clássica. Convém observar também que no caso em que a força de vínculo é elástica, a mola idealizada não corresponde à mola real no experimento discutido em [1. Ainda assim foi possível reproduzir aspectos importantes do experimento como por exemplo, as acelerações no instante inicial e a dependência das massas dos blocos da razão das acelerações. Novos e importantes avanços no problema da mola massiva foram publicados em [2] e um vídeo interessantíssimo pode ser visto no link [3].

\section{Agradecimentos}

Os autores agradecem aos árbitros pelas observações e por chamar nossa atenção para a referência [2] e para o vídeo do canal YouTube Veritassium [3].

\section{Referências}

[1] F.L. Silveira e R. Axt, Física na Escola 6, 5 (2005).

[2] R.C. Cross e M.S. Wheatland, arXiv:1208.4629 v1 (2020).

[3] https://www.youtube.com/watch?v=uiyMuHuCFo4 